\title{
Detection of PIK3R1 (L449S) Mutation in a Patient with Ovarian Cancer: A Case Report
}

\author{
Salim Moussa ${ }^{a}$ Fatima Saleh $^{a}$ Said El Shamieh ${ }^{a}$ Tarek Assi ${ }^{b, c}$ \\ Ahmad Othman ${ }^{c}$ Fadi Farhat ${ }^{b, c}$ \\ ${ }^{a}$ Faculty of Health Sciences, Beirut Arab University, Beirut, Lebanon; bepartment of \\ Hematology-Oncology, Saint Joseph Faculty of Medicine, Beirut, Lebanon; 'Department of \\ Hematology and Oncology, Hammoud Hospital UMC, Saida, Lebanon
}

\section{Keywords}

Ovarian cancer · PIK3R1 (L449S) mutation · AKT/mTOR pathway · Next-generation sequencing

\section{Abstract}

Ovarian cancer $(\mathrm{OC})$ is one of the most dangerous gynecological diseases and greatly increases the death risk worldwide. The heterogeneity of the ovarian tumors among patients and the lack of sufficient therapies for these tumors make the selection of the appropriate treatment a hard challenge. Understanding the mechanisms leading to OC becomes an urgent need in order to find out better therapeutic strategies. In this study, we have identified a point mutation (L449S) in the regulatory subunit of PI3K in an OC Lebanese patient. This genomic alteration had not been previously reported in OC and could plausibly enhance the PIK3CA amplification effect in strengthening AKT/mTOR pathway activity and leading to tumorigenesis.

(C) 2020 The Author(s).

Published by S. Karger AG, Basel

\section{Background}

Ovarian cancer (OC) is one of the most common diagnosed gynecological cancers [1], contributing $3.4 \%$ of all new cancer cases and $4.4 \%$ of deaths among women in 2018 [2]. In 2018, OC was rated as the seventh most commonly occurring cancer in women worldwide, and as the second most invasive cancer in women above the age of 40 in developed countries [3]. OC is considered the most lethal gynecological cancer [4]. If it is diagnosed at early stages, 
the 5-year survival chance becomes very high (92.3\%) compared to late stages diagnosis where it declines dramatically to $29 \%$ [5]. OC is a heterogeneous disease that differs hugely among different patients. This heterogeneity occurs at the cellular, molecular, genomic and proteomic levels thus presenting real challenges in the diagnosis and treatment of OC $[6,7]$. Ovarian epithelial tumor, also known as carcinoma, is the most common OC type accounting for more than $90 \%$ of malignant OC cases [8].

Based on histopathological, molecular and genetic alterations, ovarian epithelial tumors are classified into five main subtypes: high-grade serous (70\%), endometrioid (10\%), clear cell (10\%), mucinous (3\%) and low-grade serous OC $(<5 \%)$ [6]. Each subtype has a distinct pathogenesis, tumor progression and response to treatment; thus, it is perceived as a distinct disease [9]. High-grade serous OC is the most common and aggressive form of OC and accounts for the majority of advanced-stage cases and for most deaths from $\mathrm{OC}$ as it is the most difficult subtype to treat among all OCs [10]. In addition to the histopathological categorization, a more recent classification scheme has been developed subtyping OC into five biologically distinct subgroups; Epi-A, Epi-B, Mes, Stem-A and Stem-B; based on gene expression [11]. This subtyping might help stratify $\mathrm{OC}$ patients into subgroups for targeted therapy.

In the current study, we present the case of a woman whose final pathology was consistent with high-grade serous ovarian carcinoma involving the lymph nodes in the omentum, endometrium, cervix, ovaries, and fallopian tubes.

\section{Case Presentation}

A Lebanese 62-year-old female patient was presented to the clinic with a palpable pelvic mass and generally not feeling well. Her past medical history showed that she is a breast cancer survivor who had undergone left mastectomy with axillary lymph node dissection 13 years ago. She had MRI of the pelvis that demonstrated a large multiloculated complex cystic and solid $10 \times 10 \times 8.5 \mathrm{~cm}$ left adnexal tumoral process revealing heterogeneous hypersignal intensity on T2 with multifocal cystic loculation. Medially, a significant mass effect over the uterus was seen. The endometrium revealed heterogeneous thickening with sedimentation level and debris. Large bilateral external iliac lymph nodes were also noted on the left. Her cancer antigen 125 (CA125) was $>64.9 \mathrm{U} / \mathrm{mL}$. The possibility of endometrioid carcinoma or cyst end carcinoma was considered and thus histopathological sampling was recommended. The patient was subjected to total abdominal hysterectomy with bilateral salpingo-oophorectomy and omentectomy. The gross pathological examination showed a uterus measuring $8 \times 4 \times 4 \mathrm{~cm}$ with an attached large ovary on the left side measuring $9 \times 6 \times 6 \mathrm{~cm}$; the fallopian tube, however, could not be easily identified. On the right side, a $2.5 \times 1.6 \times 1 \mathrm{~cm}$ ovary was seen, $6 \mathrm{~cm}$ in length and $0.5 \mathrm{~cm}$ in the fallopian tube. The right ovary showed a unilocular cyst. The left ovary displayed a multicystic cut surface. The cysts showed to contain friable hemorrhagic material. The walls of the cysts were thick and rubbery; besides, the uterus had a 0.1-cm endometrium.

\section{Discussion}

The patient has undertaken the Foundation One testing, a next-generation-sequencingbased assay, in order to determine the best treatment option for her disease. The most relevant genomic findings identified were KDR, KIT, PDGFRA, PIK3CA, BRAF, KEL, PRKCI and TERC gene amplifications and PIK3R1 L449S and TP53 R273C mutations. All these alterations had been reported previously in OC except the PIK3R1 L449S mutation, a point mutation in the regulatory subunit of PI3K [12-14](Cosmic, 2019; cBioPortal, 2017). The PIK3R1 muta- 


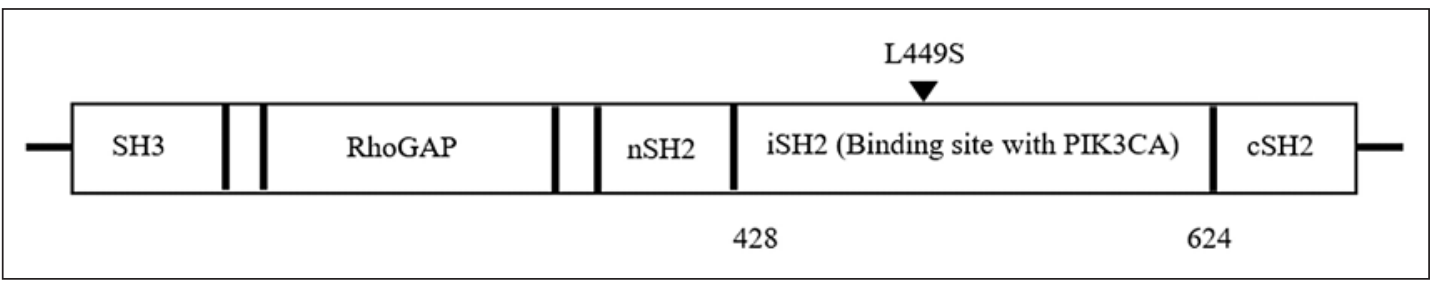

Fig. 1. Presentation of PIK3R1 (p85 $\alpha$ ) amino acid sequence depicting the mutation position of L449S at the iSH2 domain (PIK3CA binding site). iSH2: inter-SH2 domain between n-terminal and c-terminal SH2 domains. The numbers in the PIK3R1 sequence indicate the amino acid positions. $\nabla$, mutation site.

tions were observed in breast, pancreatic and colon cancers [15] and in $8.57 \%$ of ovarian carcinoma patients [16]. However, the PIK3R1 L449S substitution has been detected only in one case of breast cancer (Cosmic, 2019).

Unfortunately, there are no reportable therapeutic or clinical trial options for the PIK3R1 L449S alteration. PIK3R1 mutation was not associated with clinical benefit from either the pan-PI3K inhibitor pilaralisib or the mTOR inhibitor temsirolimus [17]. A bioinformatic pathogenicity assessment was performed using Polyphen 2 and SIFT tools and revealed that the missense L449S is probably damaging and not tolerated, respectively.

This is the first time that the PIK3R1 L449S mutation is described in OC. This alteration might be a key factor to enhance the effect of PIK3CA amplification in activating AKT/mTOR pathway and driving tumorigenesis. In most OC cases, PIK3CA (also called p110) and PIK3R1 (also called p85 $\alpha$ ) were overexpressed [12]. However, in the present case, a mutation in the inhibitory iSH2 domain of PIK3R1 was reported in coordination with PIK3CA amplification. This alteration would interrupt the binding between the catalytic and regulatory subunits PIK3CA and PIK3R1, respectively, which would stabilize PIK3CA and maintain PI3K signaling pathway activity leading to cancer development (Fig. 1). Moreover, PIK3R1 mutations destabilize PTEN (phosphatase and tensin homolog) and increase PI3K signaling [18-20].

In addition to the PIK3R1 L449S mutation, the other genomic alterations detected had certainly contributed to the cancer invasiveness. This study was limited to genomic findings due to unavailable protein data.

\section{Conclusion}

Our study is the first to show a PIK3R1 L449S mutation in OC. This alteration could be a potential factor enhancing carcinogenesis. Thus, future studies should find out a strategy to overcome the negative effect of this mutation on PI3KCA amplification and cancer development. Of interest from a therapeutic perspective, the delivery of normal PIK3R1 regulatory subunits may rescue the mutant phenotype by restoring the regulation of PIK3CA activity, which will prevent tumor growth.

\section{Statement of Ethics}

This work has been carried out in accordance with the Code of Ethics of the World Medical Association (Declaration of Helsinki). The Institutional Review board at Beirut Arab University approved this study (code: 2019H-0102-HS-R-0371). Informed consent was obtained from the patient. 


\section{Disclosure Statement}

The authors have no relevant affiliations or financial involvement with any organization or entity with a financial interest in or a financial conflict with the subject matter or materials discussed in the manuscript. This includes employment, consultancies, honoraria, stock ownership or options, expert testimony, grants or patents received or pending, or royalties. No writing assistance was utilized in the production of the manuscript.

\section{Funding Sources}

None.

\section{Author Contributions}

Salim Moussa: preparation of the manuscript; Fatima Saleh: preparation of the manuscript; Said El Shamieh: preparation and conception of the manuscript; Tarek Assi: preparation and review of the manuscript; Ahmad Othman: preparation of the manuscript; Fadi Farhat: preparation of the manuscript and final review.

\section{References}

1 Bray F, Ferlay J, Soerjomataram I, Siegel RL, Torre LA, Jemal A. Global cancer statistics 2018: GLOBOCAN estimates of incidence and mortality worldwide for 36 cancers in 185 countries. CA Cancer J Clin. 2018;68(6): 394-424.

2 Ferlay J, Colombet M, Soerjomataram I, Mathers C, Parkin DM, Piñeros M, et al. Estimating the global cancer incidence and mortality in 2018: GLOBOCAN sources and methods. Int J Cancer. 2019;144(8):1941-53.

3 Cancer Stat Facts: Ovarian Cancer[accessed 2013 Sep 7]. Available from: https: //seer.cancer.gov/statfacts/ html/ovary.html.

4 Coburn SB, Bray F, Sherman ME, Trabert B. International patterns and trends in ovarian cancer incidence, overall and by histologic subtype. Int J Cancer. 2017;140(11):2451-60.

5 Siegel RL, Miller KD, Jemal A. Cancer statistics, 2019. CA Cancer J Clin. 2019;69(1):7-34.

6 McCluggage WG. Morphological subtypes of ovarian carcinoma: a review with emphasis on new developments and pathogenesis. Pathology.2011;43(5):420-32.

7 Chen VW, Ruiz B, Killeen JL, Coté TR, Wu XC, Correa CN. Pathology and classification of ovarian tumors. Cancer. 2003; 97(S10):2631-42.

8 Sankaranarayanan R, Ferlay J. Worldwide burden of gynaecological cancer: the size of the problem. Best Pract Res Clin Obstet Gynaecol. 2006;20(2):207-25.

9 Ivanova V, Dikov T, Dimitrova N. Histologic subtypes of ovarian carcinoma: selected diagnostic and classification problems in Bulgaria: is low hospital volume an issue? Tumori. 2017;103(2):148-54.

10 Bowtell DD, Böhm S, Ahmed AA, Aspuria P-J, Bast RC Jr, Beral V, et al. Rethinking ovarian cancer II: reducing mortality from high-grade serous ovarian cancer. Nat Rev Cancer. 2015;15(11):668-79.

11 Tan TZ, Miow QH, Huang RY-J, Wong MK, Ye J, Lau JA, et al. Functional genomics identifies five distinct molecular subtypes with clinical relevance and pathways for growth control in epithelial ovarian cancer A subtyping scheme for epithelial ovarian cancer. EMBO Mol Med. 2013;5:1051-66.

12 De Marco C, Rinaldo N, Bruni P, Malzoni C, Zullo F, Fabiani F, et al. Multiple genetic alterations within the PI3K pathway are responsible for AKT activation in patients with ovarian carcinoma. PLoS One. 2013;8(2):e55362.

13 Janavičius R, Andrėkutė K, Mickys U, Rudaitis V, Brasiūnienė B, Griškevičius L. Apparently "BRCA-related" breast and ovarian cancer patient with germline TP53 mutation. Breast J. 2011;17(4):409-15.

14 Zhang L, Huang J, Yang N, Liang S, Barchetti A, Giannakakis A, et al. Integrative genomic analysis of protein kinase C (PKC) family identifies PKCiota as a biomarker and potential oncogene in ovarian carcinoma. Cancer Res. 2006;66(9):4627-35.

15 Jaiswal BS, Janakiraman V, Kljavin NM, Chaudhuri S, Stern HM, Wang W, et al. Somatic mutations in p85 $\alpha$ promote tumorigenesis through class IA PI3K activation. Cancer Cell. 2009;16(6):463-74.

16 AACR Project GENIE Consortium. AACR project GENIE: powering precision medicine through an international consortium. Cancer Discov. 2017;7(8):818-31. 
17 Matulonis U, Vergote I, Backes F, Martin LP, McMeekin S, Birrer M, et al. Phase II study of the PI3K inhibitor pilaralisib (SAR245408;XL147) in patients with advanced or recurrent endometrial carcinoma. Gynecol Oncol. 2015;136(2):246-53.

18 Cheung LWT, Hennessy BT, Li J, Yu S, Myers AP, Djordjevic B, et al. High frequency of PIK3R1 and PIK3R2 mutations in endometrial cancer elucidates a novel mechanism for regulation of PTEN protein stability. Cancer Discov. 2011;1(2):170-85.

19 Sun M, Hillmann P, Hofmann BT, Hart JR, Vogt PK. Cancer-derived mutations in the regulatory subunit p85alpha of phosphoinositide 3-kinase function through the catalytic subunit p110alpha. Proc Natl Acad Sci U S A. 2010;107(35):15547-52.

20 Urick ME, Rudd ML, Godwin AK, Sgroi D, Merino M, Bell DW. PIK3R1 (p85 $\alpha$ ) is somatically mutated at high frequency in primary endometrial cancer. Cancer Res. 2011;71(12):4061-7. 